\title{
APPLICATION OF EDDY CURRENT TECHNIQUES FOR ORBITER REINFORCED CARBON-CARBON STRUCTURAL HEALTH MONITORING
}

\author{
Buzz Wincheski $^{1}$ and John Simpson ${ }^{2}$ \\ ${ }^{1}$ NASA Langley Research Center, Hampton, VA 23681 \\ ${ }^{2}$ Lockheed Martin Space Operations, Hampton, VA 23681
}

\begin{abstract}
The development and application of advanced nondestructive evaluation techniques for the Reinforced Carbon-Carbon (RCC) components of the Space Shuttle Orbiter Leading Edge Structural Subsystem (LESS) was identified as a crucial step toward returning the shuttle fleet to service. In order to help meet this requirement, eddy current techniques have been developed for application to RCC components. Eddy current technology has been found to be particularly useful for measuring the protective coating thickness over the reinforced carbon-carbon and for the identification of near surface cracking and voids in the RCC matrix. Testing has been performed on as manufactured and flown RCC components with both actual and fabricated defects representing impact and oxidation damage. Encouraging initial results have led to the development of two separate eddy current systems for in-situ RCC inspections in the orbiter processing facility. Each of these systems has undergone blind validation testing on a full scale leading edge panel, and recently transitioned to Kennedy Space Center to be applied as a part of a comprehensive RCC inspection strategy to be performed in the orbiter processing facility after each shuttle flight.
\end{abstract}

Keywords: Eddy Current, Reinforced Carbon-Carbon, Space Shuttle

PACS: 8170.Ex, 72.80.Tm

\section{INTRODUCTION}

Following the loss of the Space Shuttle Columbia on February 1, 2003, a comprehensive inspection plan to determine the structural integrity of the Reinforced Carbon-Carbon (RCC) of the space shuttle orbiter Leading Edge Structural Subsystem (LESS) was envisioned. As recommended by the Columbia Accident Investigation Board (CAIB), advanced nondestructive evaluation techniques were sought to help meet this requirement [1]. Beginning in May 2003, the feasibility of eddy current techniques for detection of flaws in RCC was investigated at NASA Langley Research Center (LaRC). During the initial studies a State of the Art Review was performed by the Nondestructive Testing Information and Analysis Center and exploratory research was performed at NASA LaRC, Argonne National Lab, General Electric Corporate Research and Development, and Jentek Sensors [2-5]. As a result of these activities and subsequent program reviews, two systems for eddy current (EC) RCC inspections were pursued, an eddy current spot probe coupled with an automated scanning system and a Jentek Meandering Winding Magnetometer (MWM) thin film conformal array system. Both of these techniques were found to have capabilities to detect impact and oxidation damage in RCC. In addition, the techniques could measure the thickness of the protective Silicon Carbide ( $\mathrm{SiC}$ ) coating.

Following an RCC Select-down Technical Interchange Meeting (TIM) in November of 2003, development of both EC options into special test equipment for in-situ inspection of RCC on the orbiter was initiated. Although originally designed to enable full coverage scanning, a de-scope of the in-situ NDE techniques in July 2004 resulted in a decision to proceed with eddy current techniques as a local area inspection tool only. The 
EC procedures would only be applied to verify thermography indications or to check other specific areas of interest on the vehicle. In the spring of 2005, the two eddy current systems developed through these efforts were delivered to NASA Kennedy Space Center to support shuttle RCC inspections on the orbiter during normal processing flow between shuttle missions.

\section{EDDY CURRENT SYSTEMS FOR ORBITER RCC INSPECTIONS}

Figures 1 and 2 display the eddy current inspection systems developed to meet the requirements for on vehicle RCC inspection in the orbiter processing facility at Kennedy Space Center. The Jentek Meandering Winding Magnetometer system displayed in figure 1 uses a flexible array coil and spring loaded encoding wheel to produce C-scan images over a 3.7" wide section in a single pass. The system components consist of the probe head, flexible array coil, 39 channel impedance instrument, and laptop computer. Scan rates of up to 3"/sec, resulting in scan coverage of over $10 \mathrm{sq}$ in./sec have been tested without loss of image quality. The system incorporates the use of the GridStation ${ }^{\mathrm{TM}}$ method to translate coil impedance measurements into lift-off and conductivity data for the part under test, with results presented in a C-scan display [6].

In figure 2, the SAIC Ultraspect-MP system with LPS-100 manual scanner is displayed. This system consists of a high frequency eddy current surface probe connected through a spring loaded z-axis gimbal to the manual scanner. The probe is driven by the data acquisition system (DAS), which communicates with the laptop computer for acquisition of normalized probe impedance versus position. Calibration of the system is performed with a test article of nominally matching conductivity and coating thickness to the part under test, and the probe response is rotated such that lift-off is in the horizontal direction. Manual encoded scanning in two dimensions results in horizontal and vertical CScan images which are displayed on the laptop computer.

Theoretical and experimental work have shown the optimum eddy current test frequency for RCC to be between approximately $1 \mathrm{MHz}$ and $5 \mathrm{MHz}$, with lower frequencies allowing for a greater depth of penetration and higher frequencies providing

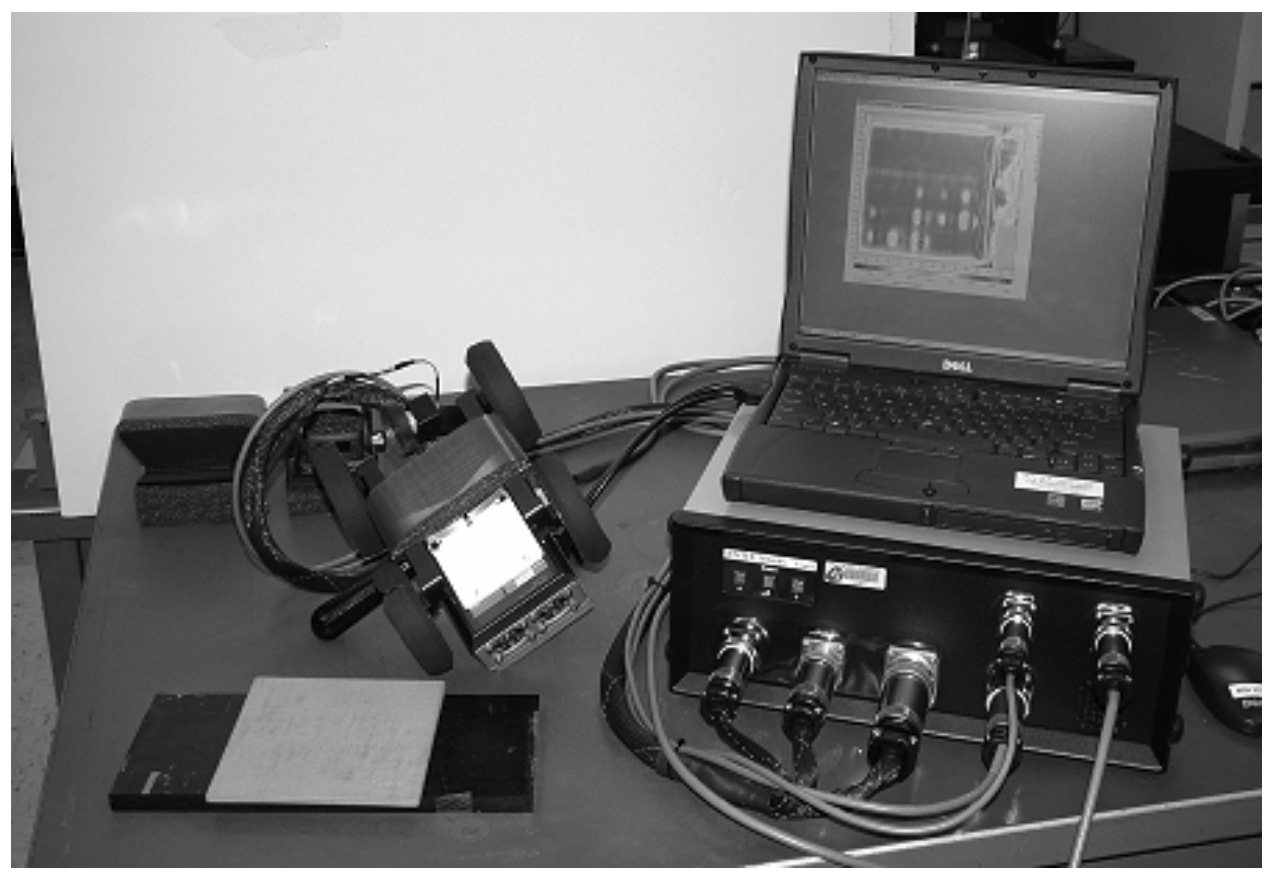

Figure 1. Jentek MWM system for in-situ eddy current inspections of RCC. 


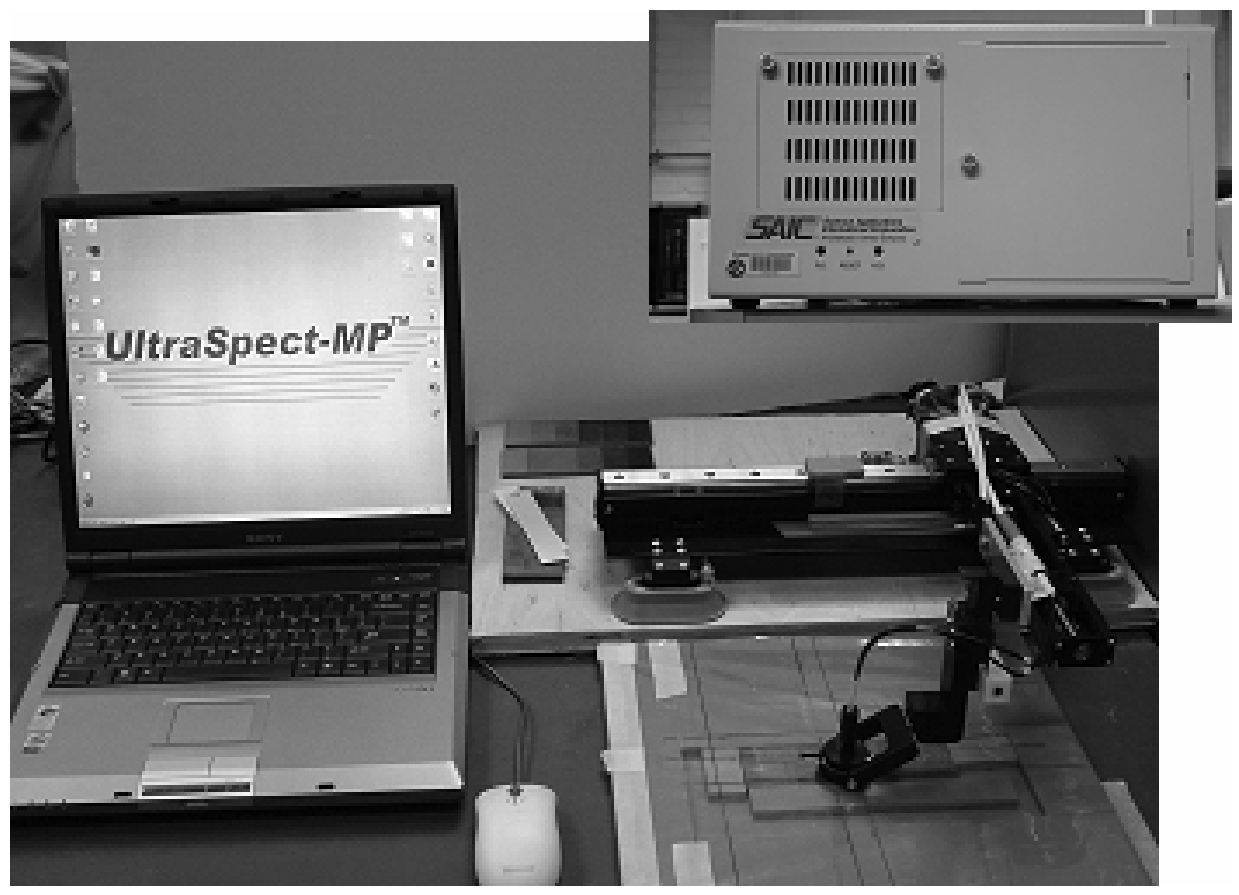

Figure 2. SAIC Ultraspect-MP eddy system for in-situ eddy current inspections of RCC.

better spatial resolution and near surface flaw detection capabilities [3-5,7]. Both the Jentek MWM and SAIC Ultraspect-MP systems support acquisition of multi-frequency data during a single scan, so that inspection for both classes of defects can be performed simultaneously.

\section{INSPECTION RESULTS ON PHYSICAL STANDARDS}

The development of the eddy current inspection systems for RCC required the use of physical standards to develop inspection procedures and determine detectable flaw sizes. To meet this requirement, newly fabricated and flown RCC material was obtained and machined to produce artifacts representative of potential failure modes of in service RCC components. This work, led by Boeing, produced several NDE reference standards designed to simulate impact and oxidation damage in RCC. The sample design and inspection results for three such standards appropriate for eddy current testing are discussed below.

The first sample design contained flat bottom holes milled from the back surface of the RCC to varying distances from the front surface. This general purpose sample could give a rough approximation for buried impact damage within RCC. Holes with diameters ranging from $1 / 8$ ” to $1 / 2$ ” were fabricated at various depths into the material. The results in Figure 3 display the $1 \mathrm{MHz}$ SAIC Ultraspect and Jentek MWM data obtained on such a flat bottom hole reference standard. The SAIC C-Scan contains the vertical channel data (perpendicular to lift-off) while the Jentek data is a conductivity C-Scan. A schematic diagram giving the location, diameter, and nominal distance from the outer surface to top of the detectable flat bottom holes is also shown in the figure. Note that the linear induction method of the MWM coil produces a characteristic dipole type pattern when scanned across the flat bottom holes while the circular spot coil attached to the SAIC system shows no angular dependence. Both eddy current techniques are seen to enable detection of flaws as small as $1 / 8$ ” diameter and as deep as 0.134 ” into the RCC.

A second sample was designed to simulate near surface oxidation damage. In this sample the SiC layer of the sample was removed so that shallow defects could be placed at 
SAIC Ultraspect

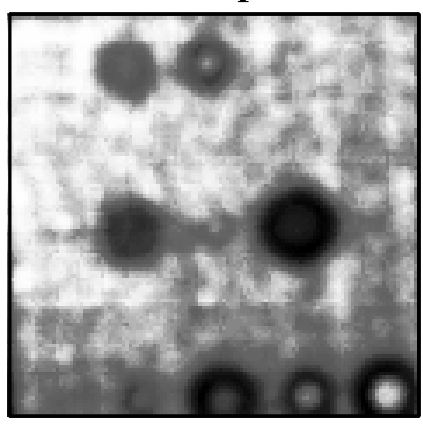

Jentek MWM

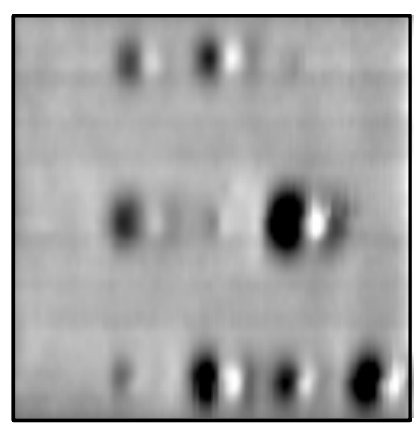

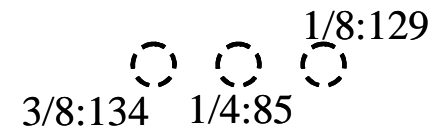

今,

$1 / 2: 131 \quad 1 / 8: 85$

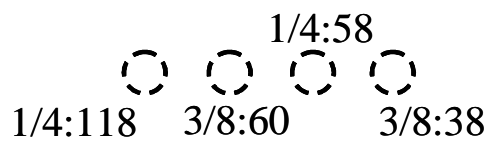

Figure 3. Eddy current inspection results for flat bottom hole standard fabricated from flown RCC material. Schematic diagram on the right displays nominal hole diameter in inches and remaining good material to unflawed front surface in mils. Inspections performed from unflawed surface.

the SiC/CC interface. As the SiC layer was destroyed in the sample fabrication, testing was performed through an insulating standoff layer used to simulate SiC. Figure 4 shows eddy current spot probe and MWM inspection results for this sample, verifying the ability to detect shallow oxidation pits directly under the $\mathrm{SiC}$ surface. A flaw map on the right side of the figure gives the diameter and depth of each of the five shallow defects in the sample. All dimensions are in inches. The displayed data are lift-off C-Scans at $5 \mathrm{MHz}$. An increase in the lift-off at the flaw locations is measured due to the loss of the conductive carbon-carbon at the SiC/CC interface which produces an increase in spacing between the test coil and the nearest conductor. The data shows that voids as small as $1 / 8$ ” diameter and 0.005 " deep can be detected with both systems. In addition to these tests with shallow pits drilled into the carbon-carbon, detection of controllably grown oxidation in RCC components has also been demonstrated [7,8].

A third eddy current standard was designed to simulate shallow near surface cracking in the carbon-carbon substrate. This sample was fabricated in a fashion similar to the oxidation sample, with the SiC layer removed. For this sample 1/2" long EDM notches were cut to depths of 0.015 ”, 0.030", 0.045" and 0.060" into the RCC. As with the oxidation standard, the $\mathrm{SiC}$ layer was destroyed in fabrication such that testing was performed through an insulating standoff layer. The MWM and SAIC inspection data for this shallow crack sample are displayed in figure 5. In this figure the MWM plots are liftoff C-scans. In the SAIC plots both the horizontal (lower figure) and vertical (upper figure) C-scans are displayed. The three deeper flaws are clearly visible, and a slight indication of the 0.015 " flaw can be detected in each data set. Due to the directionality of
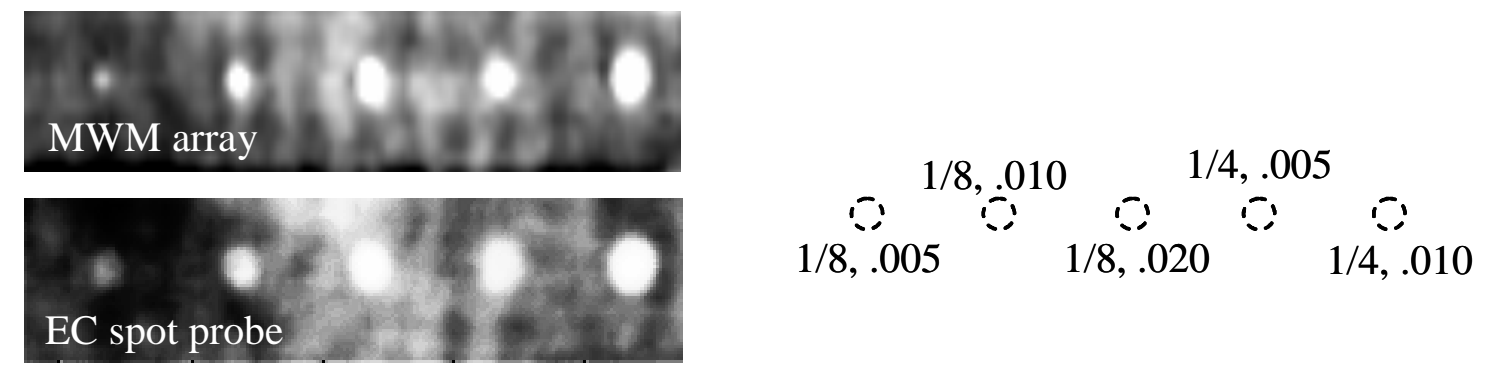

Figure 4. Inspection results and flaw map for eddy current oxidation reference standard. All dimensions are in inches. 

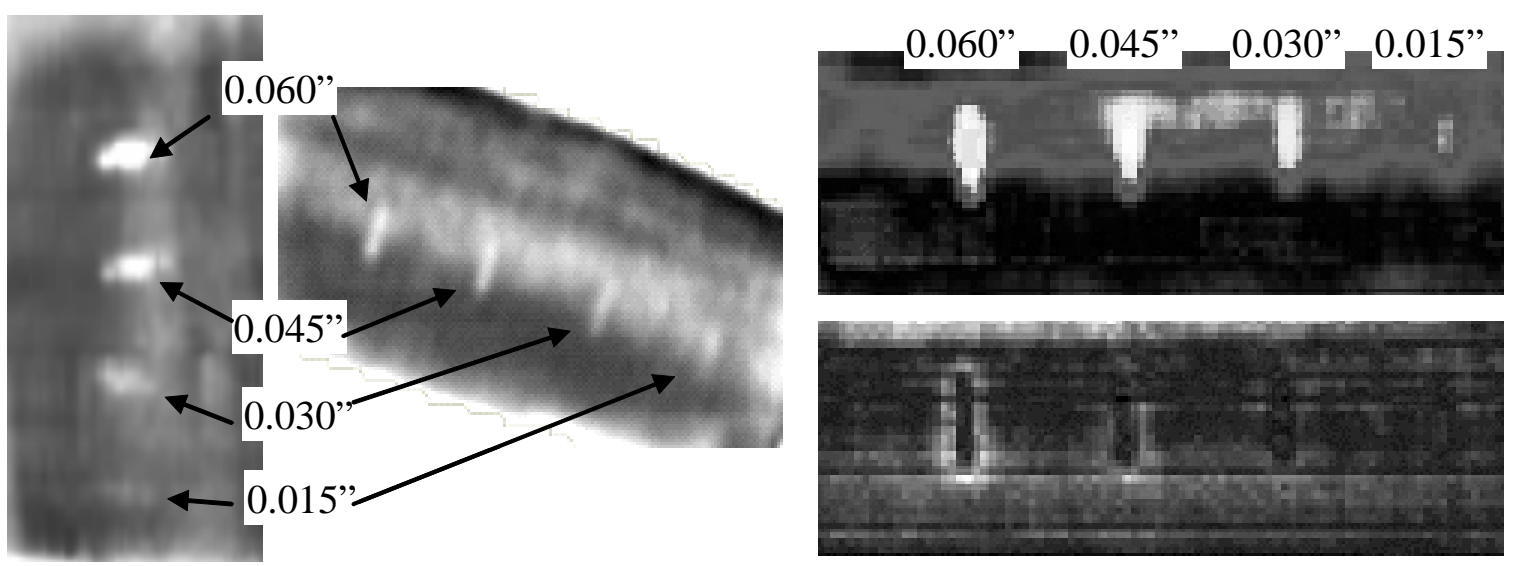

Figure 5. Inspection results for shallow EDM notch detection with Jentek MWM (left images) and SAIC Ultraspect (Right images) systems.

the MWM array, the sample was scanned in multiple directions with the Jentek system. The figure on the left displays the results when the defects are scanned with the primary coil perpendicular to the long axis of the EDM notches. The second figure shows the results when the flaws are scanned at an off-angle orientation. Although the strength of the indications are somewhat diminished in the off-angle scan, the flaws are still evident in the data.

\section{BLIND VALIDATION TESTING}

Having shown feasibility on physical test standards, a blind validation test on a full scale RCC wing leading edge panel and T-Seal was performed [9]. Artificial defects were placed in the panel from the back surface, and the panel was mounted on a stand to provide the height and orientation of an installed panel on the space shuttle orbiter. Eight individual zones, approximately 8” x 8" area each, were identified on the panel as regions of interest for eddy current inspection. In three of these zones flaw maps were provided. The location of any defects in the other four zones was hidden from the inspectors. Three regions of interest in the T-Seal were also identified for eddy current inspection. During the inspections on the panel, a 0.001" thick mylar sheet was placed over the RCC to mark a grid on the surface and protect the RCC from potential wear or contamination. Photographs of the systems in use on the full scale validation panel are shown in Figure 6.

Both the spot probe and MWM array systems performed very well during the blind
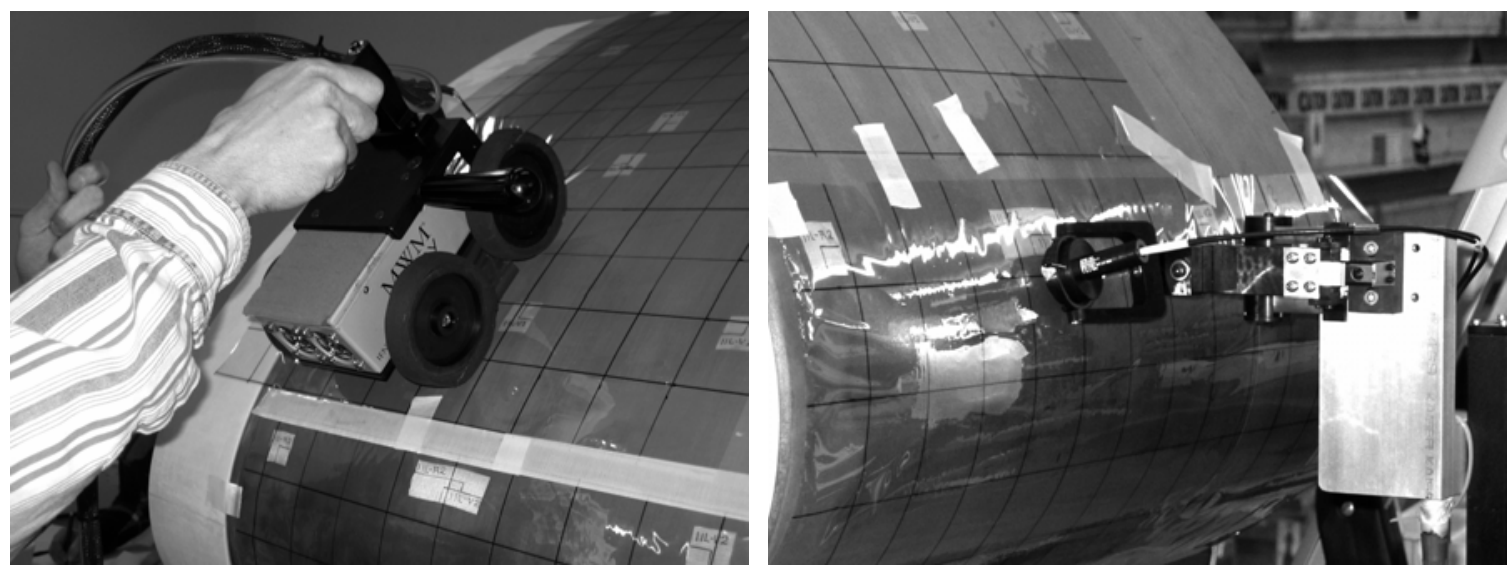

Figure 6. Inspection of full scale validation panel with MWM (left) and SAIC Ultraspect system and eddy current spot probe (right). 
validation testing. Each system detected all flaws within 0.12 ” of the outer surface. Flaws as deep as 0.173 " from the surface of the panel were also detected with both systems. Neither system reported any false calls. A probability of detection analysis performed by Sandia National Laboratory on the complete data set found the SAIC system with eddy current spot probe to have a 90\% POD for flaws 0.133 ” from the surface. The analysis of the Jentek data found the $90 \%$ POD value to be at a flaw depth of 0.155 ” from the surface.

The inspection results for one of the regions of interest are shown in figure 7 . Eight indications were reported from the SAIC inspection results. Although the dipole pattern associated with the linear induction coil of the MWM system can confuse data interpretation, a careful examination of the data shows that one additional flaw is resolved with the MWM system. The indications are labeled on the figure in order of decreasing signal level to aid in interpretation.

\section{REINFORCED CARBON CARBON HEALTH MONITORING}

The successful full scale panel validation testing results described above combined with the previous verification testing on physical standards have shown that the eddy current systems can provide useful flaw detection capabilities for orbiter RCC inspections. Detection of SiC/CC interface oxidation, shallow cracking, and internal defects buried over 1/8" deep in RCC have been demonstrated. In addition, detailed analysis of the SiC coating thickness can be performed based upon lift-off measurement of the coils over the conducting carbon-carbon substrate. In the spring of 2005 the two eddy current inspection systems described in this paper were delivered to Kennedy Space Center to provide these localized flaw detection capabilities.

Current efforts are now under way to extend the capabilities of the eddy current systems from localized flaw detection to life cycle management tools. As detailed by the Columbia Accident Investigation Board, "RCC components are weakened by mass loss caused by oxidation within the substrate, which accumulates with age. The extent of oxidation is not directly measurable, and the resulting mission life reduction is developed
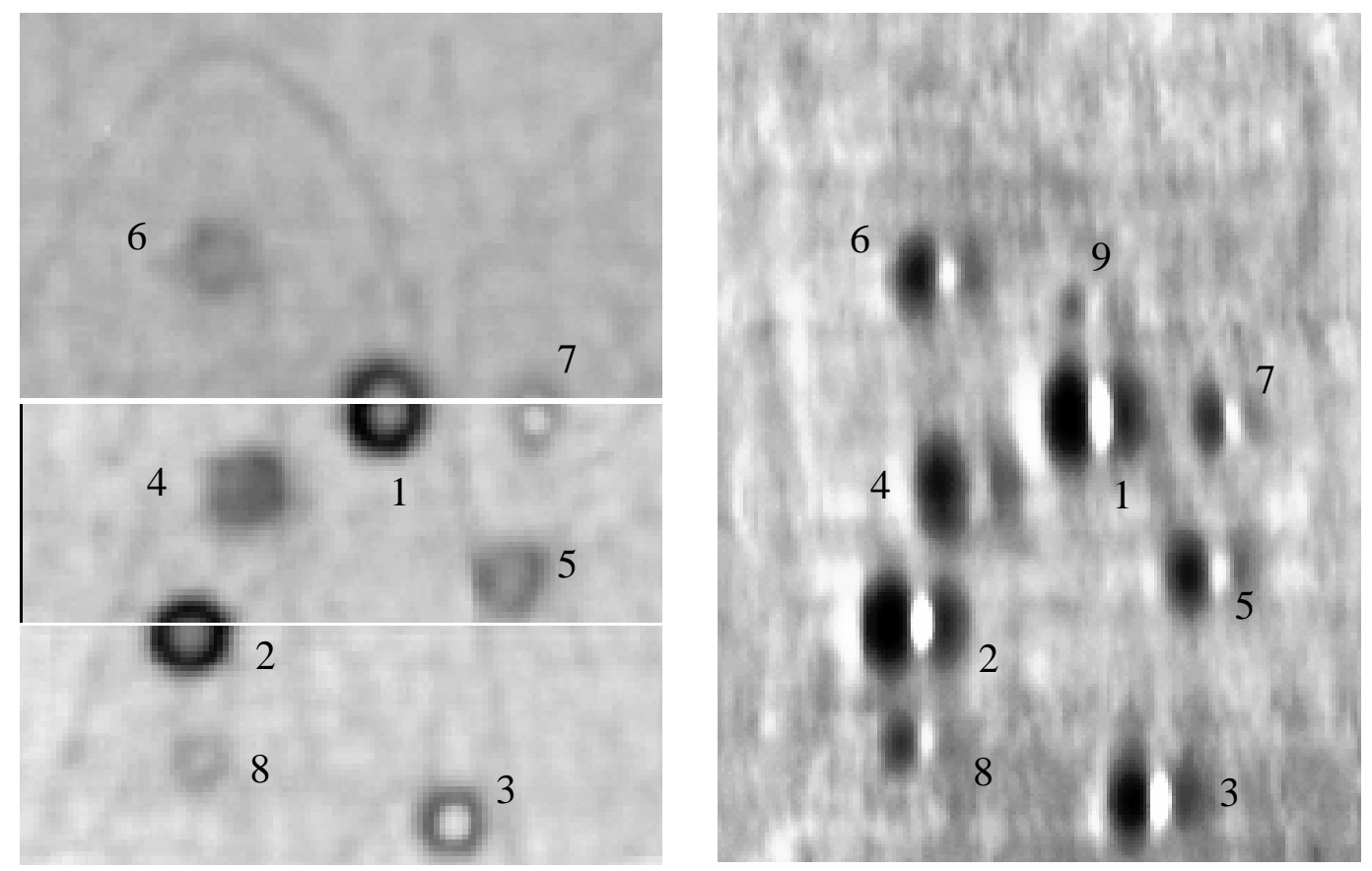

Figure 7. SAIC (left) and MWM (right) inspection results for region of interest in full panel validation tests. 
analytically." Eddy current analysis, however, provides the capability to nondestructively measure electrical conductivity of the carbon-carbon substrate. A reduction in this conductivity with mass loss is anticipated and could provide a means to directly measure the extent of RCC substrate oxidation. Initial work in this area has been promising. Measurements on a sample before and after simulated mission cycles to produce oxidation mass loss found a drop in conductivity of over $10 \%$. In addition, measurements on the full scale validation panel found a drop in conductivity of over $4 \%$ between a region of the upper panel and the apex. As the apex region of the panel experiences a higher temperature profile during re-entry, this drop in conductivity could be a measurement of increased mass loss in the apex region.

Although this preliminary work is promising, more data is required to determine background variations of the RCC conductivity, the noise floor of the eddy current conductivity measurements, and the correlation between mass loss and loss of conductivity. Experiments to determine these variables are being planned using both RCC test coupons and on orbiter tests before and after shuttle missions. An analytical study of the predicted loss in conductivity with mass loss for RCC components is also under way.

\section{SUMMARY}

Following the loss of space shuttle Columbia on February 1, 2003, the Columbia Accident Investigation Board was formed to investigate the accident and make recommendations to increase the safety of shuttle missions prior to returning the shuttle fleet to flight. One specific recommendation was to "Develop and implement a comprehensive inspection plan to determine the structural integrity of all Reinforced Carbon-Carbon system components.” This paper has presented activities associated with the development and delivery of two eddy current RCC inspection systems to help meet this requirement. Feasibility of the techniques was first demonstrated on RCC coupons, and later validated on a full scale wing leading edge RCC panel. Detection of defects including SiC/CC interface oxidation, carbon-carbon cracking, and internal voids has been

demonstrated, and the systems delivered to Kennedy Space Center to support orbiter inspections in the orbiter processing facility. Techniques for monitoring global mass loss based upon conductivity loss in the carbon-carbon are also in development in an effort to quantify global mass loss and resulting mission life reduction.

\section{ACKNOWLEDGEMENTS}

This work was conducted as part of an integrated team of participants from NASAJSC, NASA-LaRC, Sandia National Laboratories, United Space Alliance, Oceaneering Space Systems, and Boeing formed to address NDE requirements for orbiter RCC inspections.

\section{REFERENCES}

1. Columbia Accident Investigation Board Report Volume I, (August 2003).

2. Technology Assessment of Advanced Eddy Current Nondestructive Evaluation for Carbon/Carbon Composites, NTIAC Final Report, TAT No. NT-03-0002/0030, (December 2003).

3. Y. Plotnikov, Evaluation of Pulsed and Conformal EC Techniques for RCC, GE Global Research Center Final Report, (2004).

4. MWM-Array Scans of RCC Sample, Jentek Sensors Final Report, (2004). 
5. S. Bakhtiara, Multifrequency Eddy Current Inspection for Detection of Degradation in RCC Material, Argonne National Laboratory Report, (2003).

6. Goldfine, N.J, "Magnetometers for Improved Characterization in Aerospace Applications,” Materials Evaluation,Vol. 51, No. 3, 396-405, (1993).

7. B. Wincheski, Eddy Current Testing of Space Shuttle Reinforced Carbon Carbon Structures, ASNT $13^{\text {th }}$ Annual Research Symposium, Austin TX, (2004).

8. D. Roth, et al, "NDE for Characterizing Oxidation Damage in Reinforced CarbonCarbon Used on the NASA Space Shuttle Thermal Portection System," Ceramic Engineering and Science Proceedings, (2005).

9. INSitu Nondestructive Inspeciton Data Collection for Orbiter RCC Structures, NASA JSC TPS KF0520168, (March 2005). 